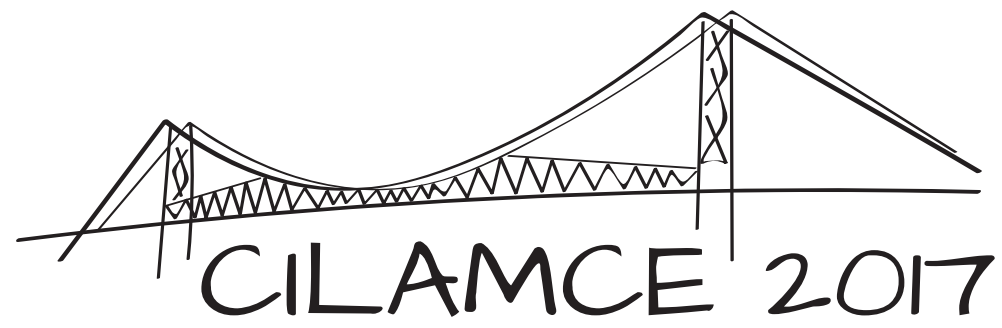

Florianópolis - Santa Catarina - Brazil

\title{
IMEX SCHEMES FOR TIME INTEGRATION OF BURGERS' EQUATION
}

\author{
Antonio M. Zarzur \\ Haroldo F. Campos Velho \\ Stephan Stephany \\ mauricio.zarzur@gmail.com \\ haroldo.camposvelho@inpe.br \\ stephan.stephany@inpe.br \\ Instituto Nacional de Pesquisas Espaciais \\ Av. dos Astronautas, 1758, 12227-010, São José dos Campos, SP, Brazil
}

\section{Saulo R. Freitas}

saulo.r.freitas@nasa.gov

NASA Goddard Space Flight Center

8800 Greenbelt Rd, 20771, Greenbelt, MD, USA

\begin{abstract}
The numerical solution of partial differential equations requires the choice of a time integration method capable of simulating the evolution of a problem. While traditional methods are usually categorized into explicit and implicit, each with their own sets of advantages and disadvantages, a more recent approach is the combination of both types into the so called IMEX schemes. These were designed to solve equations containing fast and slow time-scales in such a way that the slow terms can be solved explicitly, while the slow terms are solved implicitly, mitigating the disadvantages of each individual scheme. In this work, the finite difference approach is used to solve the viscous Burgers' equation through each of the aforementioned time integration schemes (implicit, explicit, IMEX) in a series of numerical experiments. Numerical results are evaluated against the analytical solution, and the processing time for the different schemes can be compared.
\end{abstract}

Keywords: Burgers' equation, finite differences, IMEX, numerical time integration 


\section{INTRODUCTION}

Computational simulations are based on mathematical models developed for certain natural phenomena. The solution of partial differential equations (PDEs) present in such models requires the choice of a method for time integration capable of performing a simulation while preserving numerical stability. There are methods that are more suitable to certain classes of phenomena, and therefore no single, general method can be applied to every problem. The choice must take into account not only the accuracy and stability of the method, but its computational efficiency as well.

Traditional methods are usually categorized into two families: explicit and implicit. Explicit methods are easily implemented, but their stability is conditioned by a relation between temporal and spatial discretizations, according to the Lax equivalence theorem. Such constraints may imply in very small time steps for certain problems, significantly increasing the execution time. Implicit methods, on the other hand, have no such restrictions. While the latter approach is unconditionally stable, it can also lead to computationally expensive systems of nonlinear equations that must be solved at each time step.

A more recent approach, called an IMEX (Implicit-Explicit) scheme, attempts to combine these methods to solve a particular class of problems - equations containing both fast and slow time-scales. Slow terms, which are usually nonlinear but not prone to cause instabilities, can be solved explicitly; meanwhile, the fast terms are solved implicitly. This results in a combination of different schemes that optimizes processing time by avoiding unnecessarily small time steps for the fast terms and removing the nonlinear component from the slow term. IMEX schemes have been used since the 1980s, often in conjunction with spectral methods such as in the works of Kim and Moin (1985) and Canuto et al. (1987). However, it is the classic work of Ascher et al. (1995) that first analyzes the performance of such methods and proposes improvements that will set the tone for its modern use in applications such as sea ice dynamics (Lemieux et al., 2014), wave equations (Weller et al., 2013), and radiation hydrodynamics (Kadioglu and Knoll, 2011), among many others.

This work proposes a comparative study of the three different strategies, by means of numerical experiments performed on Burgers' equation in one and two dimensions, with corresponding velocity components. Since its analytical solution is well known (Fletcher, 1983), it allows numerical experiments to be evaluated against the expected results for a given set of initial and boundary conditions.

\section{NUMERICAL TIME INTEGRATION TECHNIQUES FOR PDE}

Analytical solutions of partial differential equations, as Anderson (1996) notes, consist in closed-form expressions that represent the variation of all dependent variables continuously across the entire domain. Numerical solutions, however, can only provide values at certain discrete points along the domain (called grid points). Similarly, in the time dimension, the continuous aspect of the equation is replaced by small increments along the temporal evolution of a problem (called time steps). Simulation of any physical phenomena by this method, then, starts from a well known initial condition and integrates the discretized equations over time, generating new values at each discrete time step. 
This time integration can be done by a variety of methods, and can utilize one or more time steps to calculate the future values. In this section, the basic differences between the implicit and explicit ways of performing time integration are presented, emphasizing the disadvantages of each one. The ways in which IMEX schemes can mitigate such disadvantages is also shown.

Explicit schemes possess a single unknown variable at each grid point, $u^{n+1}$, which can be calculated using only information from previous states. Because this information is known, each grid point for time step $n+1$ can be calculated directly with no dependence on any other unknown grid points, leading to very simple computational implementations. However, these schemes must conform to CFL (Courant-Friedrichs-Lewy) stability conditions to satisfy the Lax equivalence theorem. This means that for any given value of spatial increments $(\Delta x, \Delta y)$, the time step $\Delta t$ must be smaller than a certain limit that is determined by the scheme and the problem at hand. In many cases, particularly when solving stiff systems, the required value is so small that the number of time steps required to complete the integration becomes too large. Chapra and Canale (2010) define a stiff system as one that involves both rapidly and slowly changing components.

Implicit schemes have no such restrictions, and are far more efficient at solving stiff systems (Nielsen et al., 2014). However, as they utilize the values at a grid point's neighbors at time step $n+1$ to calculate its value. Because these values are not yet known, equations need to be written for each grid point, resulting in a large system of equations that, once solved, provides values for all grid points simultaneously. In a nonlinear problem, these systems require iterative methods at each time step, greatly increasing the execution time of its numerical implementation.

IMEX schemes are a class of methods designed to solve equations that contain different terms with fast and slow time-scales. The fast terms are usually related to a linear diffusive process, which can lead to extremely small time steps in order to conform to the Lax equivalence theorem if solved explicitly (Ascher et al., 1995). It would be advantageous, then, to solve them implicitly. The slow terms, on the other hand, are possibly nonlinear in nature, which has the disadvantage of slowing down the simulation considerably if solved implicitly due to Jacobian matrix evaluations.

To solve this problem, IMEX schemes propose the solution of the fast term implicitly and the slow term explicitly. This way, it is possible to avoid unnecessarily small time steps and a situation where the solution is too complex to be processed efficiently. As noted by Ascher et al. (1995), systems such as the one described here can be written in the form of:

$$
u_{t}=f(u)+\nu g(u)
$$

where subscripts mean derivative, $f(u)$ represents the slow term and $\nu g(u)$ the fast term. Different strategies can be applied to $f$ and $g$, resulting in a new method that combines two previously unrelated schemes. It is worth noting that IMEX methods must also conform to the CFL stability conditions, such as the ones faced by explicit methods. Because the fast terms are treated implicitly, these conditions are softened, and the computational performance of such methods can be improved by using larger time steps when compared to a purely explicit scheme. If a stability condition can be reached such that the increase in time step size offsets the heavier computations per single time step, then the overall runtime can be lessened. 


\section{NUMERICAL EXPERIMENTATION: BURGERS' EQUATION}

Burgers' equation is one of the fundamental partial differential equations in fluid mechanics, as noted by Zhu et al. (2010). Initially introduced by Bateman (1915), it was later used by Burgers (1948) in his study of turbulence. Since then, it has been used to model several physical phenomena, such as shock waves, traffic flow, and acoustic waves, among many others. In its most fundamental form, with only one spatial dimension, it reads:

$$
u_{t}+u u_{x}=\nu u_{x x}
$$

where $\nu$ is the diffusion coefficient - also known as the viscosity coefficient in the context of fluid dynamics. While seemingly a simple model, Burgers' equation is nonetheless capable of modeling the interaction between nonlinear convective processes and diffusive viscous processes. It has therefore gained prominence in the testing of new numerical implementations, as noted Liu and Wang (2015).

It is possible to separate the terms of Eq. (2) in its slow (advective) and fast (diffusive) components, hereby represented by $f(u)$ and $g(u)$ respectively. In this form, the new equation reads:

$$
u_{t}=f(u)+\nu g(u)
$$

where

$$
\begin{aligned}
& f(u)=-u u_{x} \\
& g(u)=u_{x x}
\end{aligned}
$$

and mirrors the canonical IMEX form described by Eq. (1), making it a suitable candidate to test this type of integration.

When considering two spatial dimensions $(x, y)$ and two velocity components $(u, v)$, Burgers' equation becomes a system of two coupled PDEs, which are essentially a simplification of the Navier Stokes equations (Srivastava et al., 2011):

$$
\begin{aligned}
& u_{t}+u u_{x}+v u_{y}=\nu\left(u_{x x}+u_{y y}\right) \\
& v_{t}+u v_{x}+v v_{y}=\nu\left(v_{x x}+v_{y y}\right)
\end{aligned}
$$

As with the one dimensional case, it is possible to rewrite Eq. (6) and Eq. (7) into a single equation in matrix form that matches the IMEX form:

$$
\Phi_{t}=f(\Phi)+\nu g(\Phi)
$$

where $\Phi$ is the velocity vector with components $u$ and $v$, and functions $f$ and $g$ are given by:

$$
\begin{aligned}
& f(\Phi)=-\frac{1}{2} \nabla(\Phi \cdot \Phi) \\
& g(\Phi)=\nabla^{2} \Phi
\end{aligned}
$$

In order to compare the three different time integration strategies - explicit, implicit, and IMEX - a series of discretization schemes for Burgers' equation both in its one-dimensional 
and two-dimensional forms were implemented in this work. Spatial discretizations can be handled by many different methods; however, this work will focus only on the finite differences approach, using second order approximations for the derivatives. Accordingly, discretizations for the one-dimensional spatial derivatives are given by:

$$
\begin{aligned}
& u_{x} \approx \frac{u_{i+1}^{n}-u_{i-1}^{n}}{2 \Delta x} \\
& u_{x x} \approx \frac{u_{i+1}^{n}-2 u_{i}^{n}+u_{i-1}^{n}}{\Delta x^{2}}
\end{aligned}
$$

where $u_{i}^{n}$ denotes $u\left(x_{i}, t_{n}\right)$.

Similarly, discretizations for the two-dimensional spatial derivatives are given by:

$$
\begin{aligned}
& u_{x} \approx \frac{u_{i+1, j}^{n}-u_{i-1, j}^{n}}{2 \Delta x} \\
& u_{y} \approx \frac{u_{i, j+1}^{n}-u_{i, j-1}^{n}}{2 \Delta y} \\
& u_{x x} \approx \frac{u_{i+1, j}^{n}-2 u_{i, j}^{n}+u_{i-1, j}^{n}}{\Delta x^{2}} \\
& u_{y y} \approx \frac{u_{i, j+1}^{n}-2 u_{i, j}^{n}+u_{i, j-1}^{n}}{\Delta y^{2}}
\end{aligned}
$$

where $u_{i, j}^{n}$ denotes $u\left(x_{i}, y_{j}, t_{n}\right)$.

\subsection{Time integration schemes for Burgers' equation}

A simple explicit integration scheme can be achieved by utilizing a forward-Euler method for temporal component, and second order central differences for the spatial components. The general form for this scheme, known as FTCS (Forward-Time, Central-Space), reads:

$$
\frac{u^{n+1}-u^{n}}{\Delta t}=f\left(u^{n}\right)+\nu g\left(u^{n}\right)
$$

Srivastava et al. (2011) show that Burgers' equation can be integrated implicitly using the Crank-Nicolson scheme. The scheme reads:

$$
\frac{u^{n+1}-u^{n}}{\Delta t}=\frac{1}{2}\left[f\left(u^{n+1}\right)+f\left(u^{n}\right)\right]+\frac{\nu}{2}\left[g\left(u^{n+1}\right)+g\left(u^{n}\right)\right] .
$$

In the one-dimensional case, this implicit discretization generates a system of $n_{x}$ nonlinear equations that must be solved at each time step, where $n_{x}$ is the number of grid points along the spatial axis. For this work, the iterative Newton-Raphson method is used to approximate the solution of the nonlinear system after an evaluation of its associated Jacobian matrix. In two dimensions, the system contains $2 n_{x} n_{y}$ equations, where $n_{y}$ is the number of grid points along the $y$ axis, because the two velocity components must be solved simultaneously.

Durran and Blossey (2012) propose a series of multistep IMEX methods categorized into families. These are general formulations that allows the matching of different strategies via a set 
of parameters. This work implements the family based on the combination of Adams implicit and Adams-Bashforth explicit schemes. Equation (19) shows the integration strategy for the implicit term, and Eq. (20) the one for the explicit term:

$$
\begin{aligned}
& g(u) \approx \frac{(1+c)}{2} g\left(u^{n+1}\right)+\frac{(1-2 c)}{2} g\left(u^{n}\right)+\frac{c}{2} g\left(u^{n-1}\right) \\
& f(u) \approx \frac{(3+b)}{2} f\left(u^{n}\right)-\frac{(1+2 b)}{2} f\left(u^{n-1}\right)+\frac{b}{2} f\left(u^{n-2}\right)
\end{aligned}
$$

Therefore, the generalized time stepping scheme for Burgers' equation based on this family of IMEX methods is given by:

$$
\begin{aligned}
\frac{u^{n+1}-u^{n}}{\Delta t}=\frac{(3+b)}{2} f\left(u^{n}\right) & -\frac{(1+2 b)}{2} f\left(u^{n-1}\right)+\frac{b}{2} f\left(u^{n-2}\right) \\
& +\nu\left[\frac{(1+c)}{2} g\left(u^{n+1}\right)+\frac{(1-2 c)}{2} g\left(u^{n}\right)+\frac{c}{2} g\left(u^{n-1}\right)\right]
\end{aligned}
$$

where the choice of parameters $b$ and $c$ defines the resulting multistep IMEX scheme.

Two variations of this family of methods were implemented. In the first, the choice of parameters $b=\frac{5}{6}$ and $c=\frac{1}{2}$ results in a combination of a two-step Adams-Moulton scheme for $g(u)$ and a three-step Adams-Bashforth explicit scheme for $f(u)$. This implementation will be henceforth referred to as AM2*-AB3 in order to maintain consistency with the nomenclatures used by Fornberg and Driscoll (1999) and Durran and Blossey (2012). Equation (21) then becomes:

$$
\frac{u^{n+1}-u^{n}}{\Delta t}=\frac{23}{12} f\left(u^{n}\right)-\frac{4}{3} f\left(u^{n-1}\right)+\frac{5}{12} f\left(u^{n-2}\right)+\nu\left[\frac{3}{4} g\left(u^{n+1}\right)+\frac{1}{4} g\left(u^{n-1}\right)\right]
$$

The second scheme is similar to Eq. (22), but uses a three-step Adams implicit scheme. To achieve this, parameters $b=\frac{5}{6}$ and $c=\frac{3}{2}$ are chosen. This method is hereby called AI $2 *-\mathrm{AB} 3$, and its general form is given by:

$$
\begin{aligned}
\frac{u^{n+1}-u^{n}}{\Delta t}=\frac{23}{12} f\left(u^{n}\right)-\frac{4}{3} f\left(u^{n-1}\right)+\frac{5}{12} f & \left(u^{n-2}\right) \\
& +\nu\left[\frac{5}{4} g\left(u^{n+1}\right)-g\left(u^{n}\right)+\frac{3}{4} g\left(u^{n-1}\right)\right]
\end{aligned}
$$

The schemes discussed in this section were implemented for the one-dimensional case, described by Eq. (3), as well as the two-dimensional case described by Eq. (8). Because IMEX schemes treat the nonlinear term explicitly, the resulting system of equations is linear and thus much simpler to solve. In two dimensions, it has the added effect of uncoupling the solutions for $u$ and $v$ at the time step level.

In order to complete the discretization of Burgers' equation, finite difference operators are applied to the spatial variables. In the one-dimensional case, the derivatives in Eqs. (4 - 5) are 
replaced using approximations given by Eqs. $(11-12)$, whereas the derivatives resulting from Eqs. $(9-10)$ are replaced using approximations given by Eqs. $(13-16)$. This provides second order approximations for both the advection and diffusion terms.

The temporal $(\Delta t)$ and spatial $(\Delta x, \Delta y)$ increments in the numerical experiments were chosen to ensure the stability of the proposed methods at first. As the spatial resolution and viscosity increase, but the temporal increment remains fixed, stability is pushed to its limits until the method is no longer stable. To solve this problem, a smaller value of $\Delta t$ would need to be picked. The objective of this strategy is to show empirically that the proposed IMEX schemes remain stable at higher resolutions than the fully explicit method. This means that, given a fixed resolution, a larger value of $\Delta t$ can be used to integrate the problem using an IMEX method, which in turn decreases the number of overall time steps required to complete the simulation. While smaller time increments would in theory provide higher accuracy, Mesinger and Arakawa (1976) show that errors in spatial discretization far outweigh those originating from time discretization, and therefore grid refinements provide better gains in accuracy than smaller $\Delta t$.

\section{NUMERICAL EXPERIMENT IN ONE DIMENSION}

In this section, the accuracy of the proposed methods in solving the one-dimensional Burgers' equation is evaluated via a test case that has already been presented in in the literature.

Problem 1. Nielsen et al. (2014) show that an exact solution to the one-dimensional Burgers' equation, representing a wave that propagates left-to-right as time advances, is given by:

$$
u(x, t)=1-\tanh \left(\frac{x-t}{2 \nu}\right)
$$

The first test case considers a spatial domain bounded by $x \in[-10,10]$ with initial conditions given by Eq. (24) at $t=0$, and boundary conditions given by the same equation at each time step of $\Delta t=10^{-3}$. For this test case, the viscosity coefficient chosen is $\nu=0.0625$. The simulation is considered over at $t=5$ s, i.e. 5000 time steps.

Table 1 shows the error given by the $L^{1}$ norm of $u$, taken at $t=5 \mathrm{~s}$, for the different methods and for a variety of grid sizes. For finer grids, such as $n_{x}=2000$, the explicit scheme is no longer within its stability conditions, and thus its error is not shown.

The second test case employs the same initial and boundary conditions, and the same timestep of $\Delta t=10^{-3}$, but with a higher viscosity coefficient $\nu=0.5$, in order to evaluate how the implicit treatment of the diffusion term affects accuracy. The results are presented in Table 2.

Figure 1 shows that the solution obtained with the AM2*-AB3 scheme at three different integration times ( $t=0, t=2.5, t=5 \mathrm{~s})$, using a 500-point grid, is consistent with those obtained by Nielsen et al. (2014), giving further confirmation of the proposed method's capacity to simulate the problem.

Figure 2 shows a close-up on the wave front at $t=5 \mathrm{~s}$, comparing the analytical solution to that given by the AI2*-AB3 IMEX scheme using a 500-point grid, and showing that both solutions are practically superposed. 
Table 1: Error given by the $L^{1}$ norm in $u$ for the different methods and for various grid sizes in Problem 1, using $\nu=0.0625$ and $\Delta t=10^{-3}$

\begin{tabular}{ccccc}
\hline Grid Size & FTCS & Crank-Nicolson & AI2*-AB3 & AM2*-AB3 \\
\hline 100 & 2.23521 E-002 & 2.19635 E-002 & 2.19647 E-002 & 2.19640 E-002 \\
125 & 1.14021 E-002 & 1.11344 E-002 & 1.11360 E-002 & 1.11350 E-002 \\
250 & 1.79243 E-003 & 1.72960 E-003 & 1.72987 E-003 & 1.72967 E-003 \\
500 & 4.34950 E-004 & 3.78677 E-004 & 3.78789 E-004 & 3.78692 E-004 \\
1000 & 1.53812 E-004 & 9.17426 E-005 & 9.18667 E-005 & 9.17651 E-005 \\
2000 & N/A & 2.28338 E-005 & 2.29382 E-005 & 2.28461 E-005 \\
\hline
\end{tabular}

Table 2: Error given by the $L^{1}$ norm in $u$ for the different methods and for various grid sizes in Problem 1, using $\nu=0.5$ and $\Delta t=10^{-3}$

\begin{tabular}{ccccc}
\hline Grid Size & FTCS & Crank-Nicolson & AI2*-AB3 & AM2*-AB3 \\
\hline 100 & 1.14792 E-003 & 1.10247 E-003 & 1.10114 E-003 & 1.10113 E-003 \\
125 & 7.51261 E-004 & 7.01639 E-004 & 7.00102 E-004 & 7.00092 E-004 \\
250 & 2.29472 E-004 & 1.76976 E-004 & 1.75044 E-004 & 1.75030 E-004 \\
500 & 1.04186 E-004 & 4.59543 E-005 & 4.38312 E-005 & 4.38180 E-005 \\
1000 & N/A & 1.31947 E-005 & 1.09838 E-005 & 1.09710 E-005 \\
2000 & N/A & 5.01417 E-006 & 2.76501 E-006 & 2.75213 E-006 \\
\hline
\end{tabular}

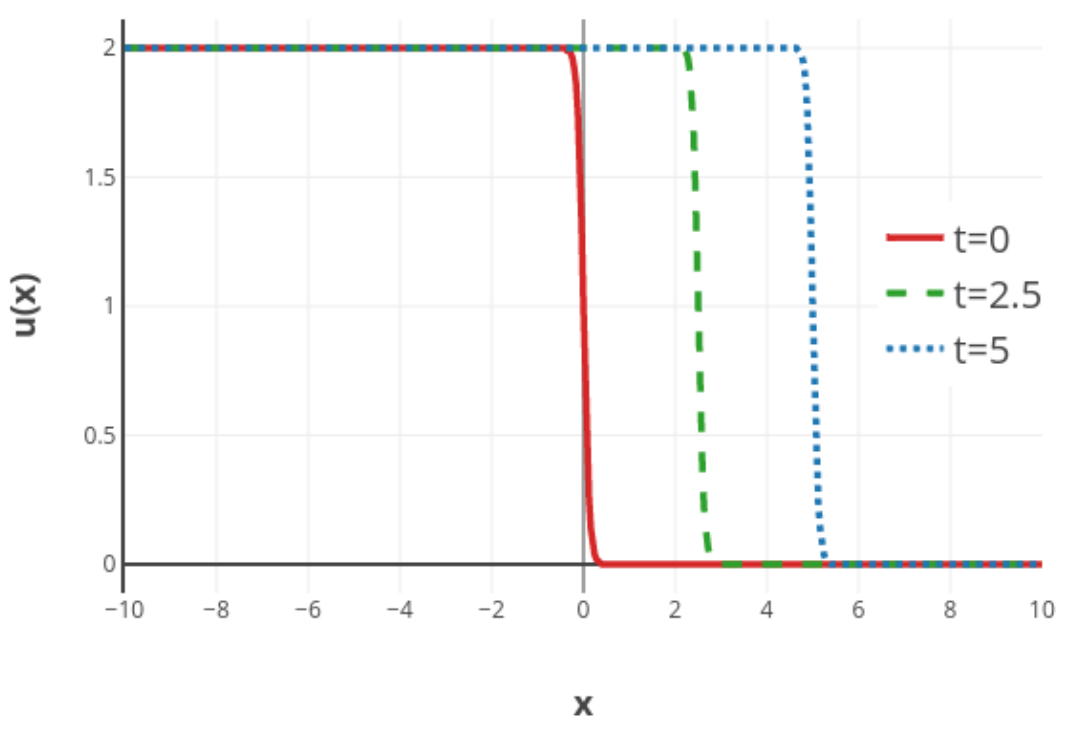

Figure 1: Solution of problem 1 using the $\mathrm{AM} 2 *-\mathrm{AB} 3$ scheme at three different integration times 


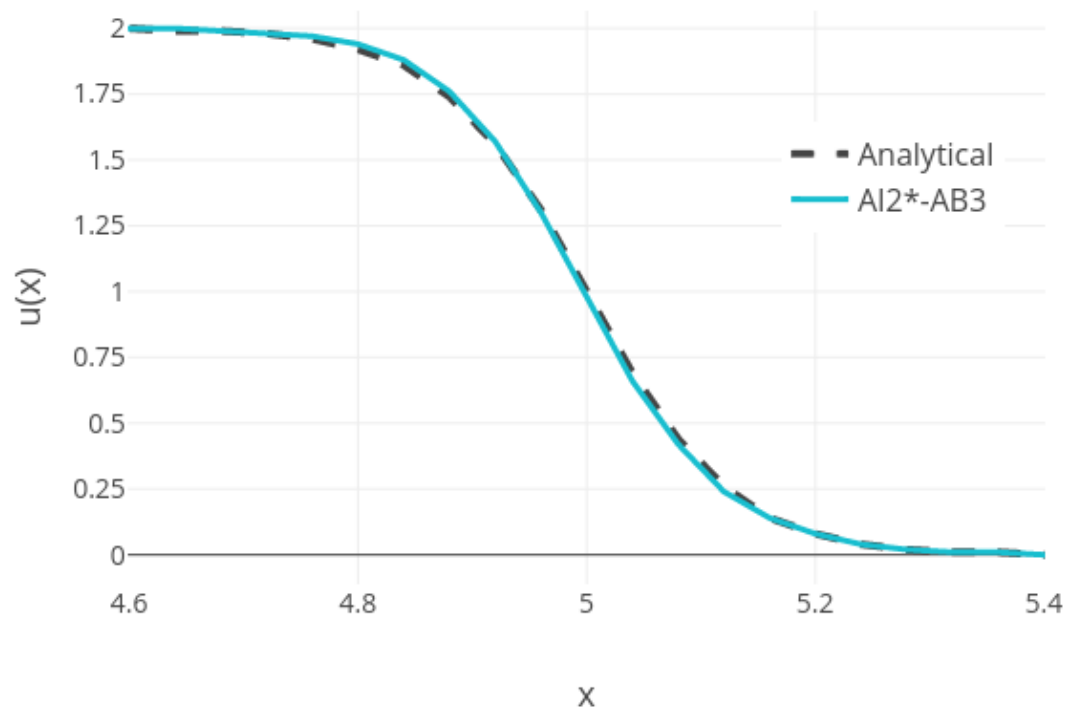

Figure 2: Close up of wave front given by the analytical and $\mathrm{AI} 2 *-\mathrm{AB} 3$ solution at $\mathrm{t}=5 \mathrm{~s}$

The experiment shows that both IMEX schemes are able to simulate the problem with accuracy comparable to a purely implicit scheme, with the advantage of the nonlinear term being treated explicitly. It also shows that they can maintain stability even in certain regions where the explicit scheme can no longer be reliable.

\section{NUMERICAL EXPERIMENTS IN TWO DIMENSIONS}

In this section, the accuracy of the proposed methods for solving the two-dimensional Burgers' equation is evaluated. Two different test cases were chosen, both already presented in the literature, in order to assess the performance of the proposed implementation.

Problem 2. The exact solutions obtained by Fletcher (1983) and tested by Zhu et al. (2010), given by Eq. (25) and Eq. (26), are used to generate initial (taken at $t=0$ ) and boundary conditions for the spatial domain $x \in[0,1], y \in[0,1]$.

$$
\begin{aligned}
& u(x, y, t)=\frac{3}{4}-\frac{1}{4\left(1+e^{(-t-4 x+4 y) /(32 \nu)}\right)} \\
& v(x, y, t)=\frac{3}{4}+\frac{1}{4\left(1+e^{(-t-4 x+4 y) /(32 \nu)}\right)}
\end{aligned}
$$

The first test is taken directly from the work of Zhu et al. (2010), in order to evaluate the accuracy of the implementation. A viscosity coefficient $\nu=\frac{1}{80}$ is used, and the equation is integrated from $t=0$ to $t=0.5 \mathrm{~s}$ using a time step of $0.00025 \mathrm{~s}$ (i.e.: 2000 time steps). Table 3 shows the error given by the $L^{1}$ norm of $u$, taken at $t=0.5 \mathrm{~s}$, for each implemented method. The corresponding solution of $u$ using the AI $2 *-\mathrm{AB} 3$ scheme is shown in Figure 3 at three different stages: (a) at $t=0$, showing the initial conditions to the problem; (b) an intermediary solution at $t=0.25 \mathrm{~s}$, after 1000 time steps; and (c) the end of the integration at $t=0.5 \mathrm{~s}$. Table 4 shows the same error metrics for $v$, and the corresponding solution by the AI2*-AB3 scheme is shown in Figure 4. 
Table 3: Error given by the $L^{1}$ norm in $u$ for each method in solving Problem 2

\begin{tabular}{ccccc}
\hline Grid Size & FTCS & Crank-Nicolson & AI2*-AB3 & AM2*-AB3 \\
\hline $10 \times 10$ & $9.76529 \mathrm{E}-004$ & $9.74884 \mathrm{E}-004$ & $9.74886 \mathrm{E}-004$ & $9.74885 \mathrm{E}-004$ \\
$20 \times 20$ & $2.38956 \mathrm{E}-004$ & $2.37644 \mathrm{E}-004$ & $2.37646 \mathrm{E}-004$ & $2.37645 \mathrm{E}-004$ \\
$30 \times 30$ & $1.04980 \mathrm{E}-004$ & $1.03780 \mathrm{E}-004$ & $1.03781 \mathrm{E}-004$ & $1.03781 \mathrm{E}-004$ \\
$40 \times 40$ & $5.94381 \mathrm{E}-005$ & $5.81135 \mathrm{E}-005$ & $5.81147 \mathrm{E}-005$ & $5.81144 \mathrm{E}-005$ \\
$50 \times 50$ & $3.85050 \mathrm{E}-005$ & $3.71850 \mathrm{E}-005$ & $3.71861 \mathrm{E}-005$ & $3.71857 \mathrm{E}-005$ \\
\hline
\end{tabular}

(a)

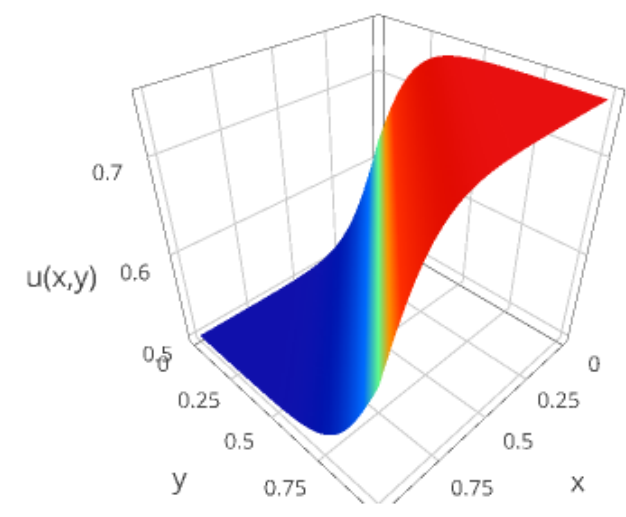

(c) (b)
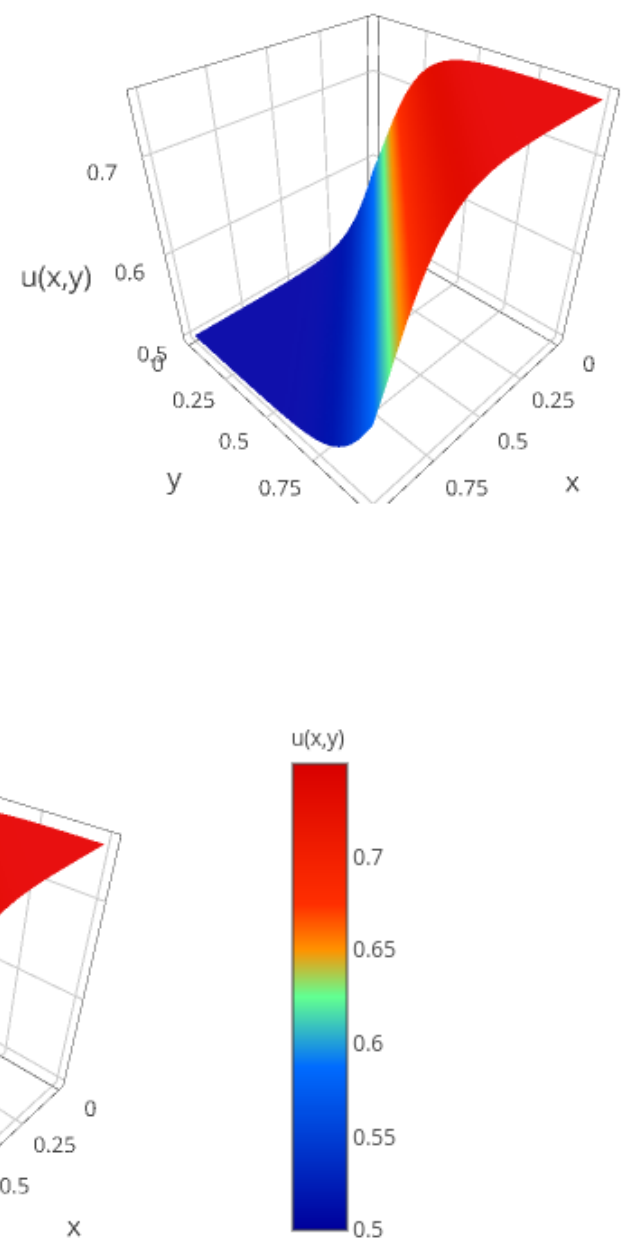

Figure 3: Solution of $u(x, y, t)$ for Problem 2 at $(a) t=0$, (b) $t=0.25 s$, and (c) $t=0.5 s$ using AI2*-AB3 
Table 4: Error given by the $L^{1}$ norm in $v$ for each method in solving Problem 2

\begin{tabular}{ccccc}
\hline Grid Size & FTCS & Crank-Nicolson & AI2*-AB3 & AM2*-AB3 \\
\hline $10 \times 10$ & 9.76529 E-004 & 9.74884 E-004 & 9.74886 E-004 & 9.74885 E-004 \\
$20 \times 20$ & 2.38956 E-004 & 2.37644 E-004 & 2.37646 E-004 & 2.37645 E-004 \\
$30 \times 30$ & 1.04980 E-004 & 1.03780 E-004 & 1.03781 E-004 & 1.03781 E-004 \\
$40 \times 40$ & 5.94381 E-005 & 5.81135 E-005 & 5.81147 E-005 & 5.81144 E-005 \\
$50 \times 50$ & 3.85050 E-005 & 3.71850 E-005 & 3.71861 E-005 & 3.71857 E-005 \\
\hline
\end{tabular}

(a)

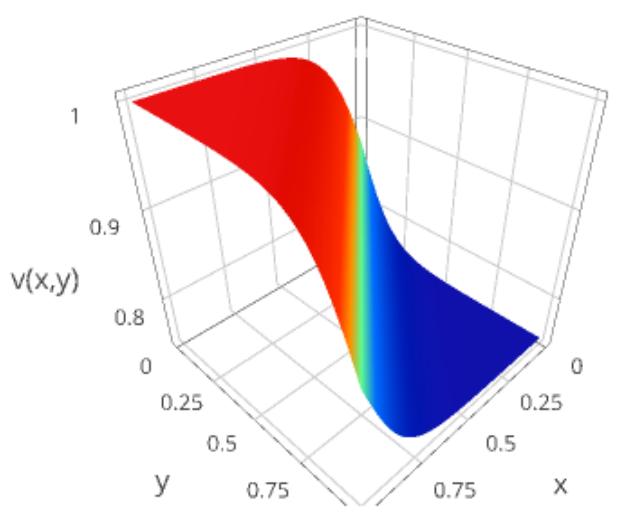

(c) (b)
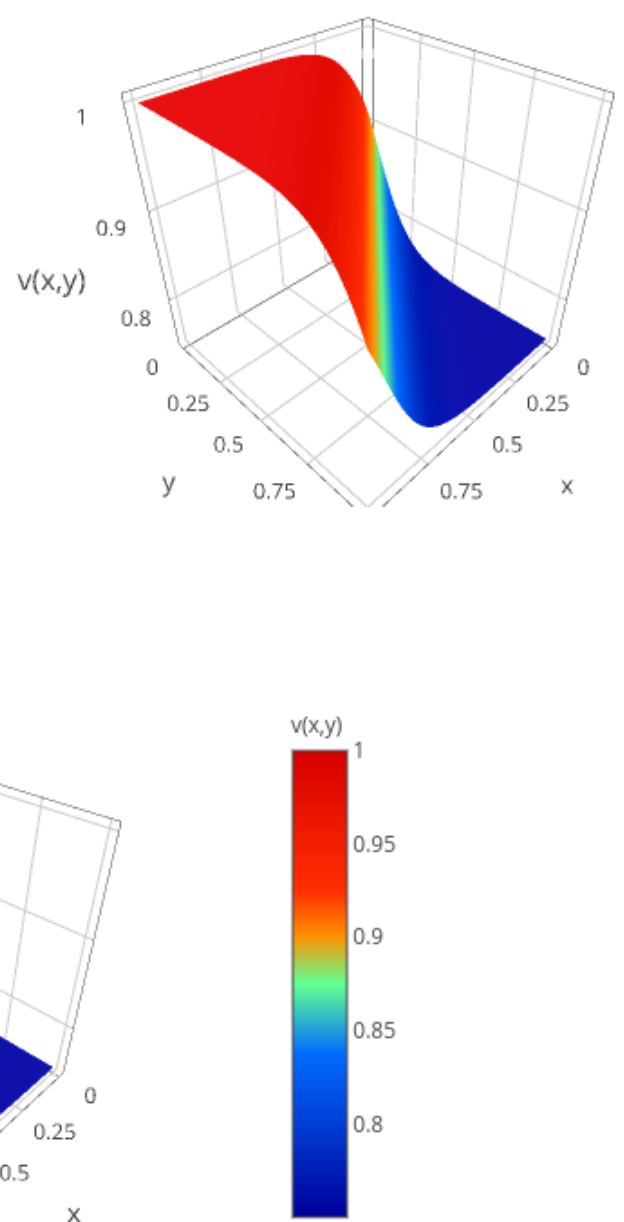

Figure 4: Solution of $v(x, y, t)$ for Problem 2 at $(a) t=0$, (b) $t=0.25 s$, and $(c) t=0.5 s$ using AI2*-AB3

Both IMEX methods closely match the accuracy of the fully implicit method for this problem, providing a solution consistent with results obtained by the authors via a different implementation. This confirms that the proposed schemes are capable of solving the studied test case. 
It should be noted that, given the precision adopted in this work, Tables 3 and 4 look identical. This illusion is caused by the similarity of the initial conditions, which makes the difference between errors so small as to only show up in the 12th to 14th decimal, or in this case E-016 to E-018. In practice, the difference is so close to machine zero that for all intents and purposes the error is considered identical.

Problem 3. The exact solutions, obtained by Kweyu et al. (2012) via Hopf-Cole transformation, are given by Eq. (27) and Eq. (28). These are used to generate initial (taken at $t=0$ ) and boundary conditions for the spatial domain $x \in[0,1], y \in[0,1]$.

$$
\begin{aligned}
& u(x, y, t)=\nu\left[\frac{-2 y-2 \pi e^{-2 \nu \pi^{2} t} \sin (\pi y)(\cos (\pi x)-\sin (\pi x))}{100+x y+e^{-2 \nu \pi^{2} t} \sin (\pi y)(\cos (\pi x)-\sin (\pi x))}\right] \\
& v(x, y, t)=\nu\left[\frac{-2 x-2 \pi e^{-2 \nu \pi^{2} t} \cos (\pi y)(\cos (\pi x)+\sin (\pi x))}{100+x y+e^{-2 \nu \pi^{2} t} \sin (\pi y)(\cos (\pi x)-\sin (\pi x))}\right]
\end{aligned}
$$

Two experiments were made using this set of initial and boundary conditions. The first, taken directly from the work developed by Kweyu et al. (2012), uses a very low viscosity coefficient $(\nu=1 / 4000)$. This test case was chosen to verify the algorithms presented here and show that the results are consistent with the literature. Table 5 and Table 6 show the error of each method given by the $L^{1}$ norm for $u$ and $v$, respectively, taken at $t=1 \mathrm{~s}$ using a time step of $10^{-3} s$.

Table 5: Error of $u$ in solving Problem 3 with each proposed method, using $\nu=1 / 4000$ and $\Delta t=10^{-3}$

\begin{tabular}{ccccc}
\hline Grid Size & FTCS & Crank-Nicolson & AI2*-AB3 & AM2*-AB3 \\
\hline $4 \times 4$ & 1.37267 E-009 & 1.37273 E-009 & 1.37273 E-009 & 1.37273 E-009 \\
$8 \times 8$ & 4.58934 E-010 & 4.59020 E-010 & 4.59020 E-010 & 4.59020 E-010 \\
$16 \times 16$ & 1.27003 E-010 & 1.27100 E-010 & 1.27101 E-010 & 1.27100 E-010 \\
$32 \times 32$ & 3.29537 E-011 & 3.30561 E-011 & 3.30559 E-011 & 3.30559 E-011 \\
$64 \times 64$ & 8.27575 E-012 & 8.37981 E-012 & 8.37961 E-012 & 8.37960 E-012 \\
\hline
\end{tabular}

The results obtained via the FTCS and Crank-Nicolson schemes match those reported by the authors for the same test case. Furthermore, the IMEX schemes show accuracy in the same order of both methods. The similarities between the results are to be expected, due to the very low viscosity coefficient chosen by the authors - the contribution from $g(u)$ is so small that its implicit treatment has little to no effect.

That being the case, another experiment was proposed, using a more significant diffusion coefficient $\nu=0.5$ with the same initial and boundary conditions from Eq. (27) and Eq. (28). In this case, the increased viscosity renders the FTCS scheme unstable as the spatial resolution increases past a certain point, as it also factors in the CFL stability condition. The error of $u$ and $v$ at time $t=1 \mathrm{~s}$, given by the $L^{1}$ norm, is shown in Table 7 and Table 8 , respectively. 
Table 6: Error of $v$ in solving Problem 3 with each proposed method, using $\nu=1 / 4000$ and $\Delta t=10^{-3}$

\begin{tabular}{ccccc}
\hline Grid Size & FTCS & Crank-Nicolson & AI2*-AB3 & AM2*-AB3 \\
\hline $4 \times 4$ & 7.73967 E-010 & 7.74001 E-010 & 7.74001 E-010 & 7.74000 E-010 \\
$8 \times 8$ & 3.55949 E-010 & 3.56016 E-010 & 3.56016 E-010 & 3.56016 E-010 \\
$16 \times 16$ & 1.10927 E-010 & 1.11013 E-010 & 1.11013 E-010 & 1.11013 E-010 \\
$32 \times 32$ & 3.02421 E-011 & 3.03372 E-011 & 3.03371 E-011 & 3.03370 E-011 \\
$64 \times 64$ & 7.73355 E-012 & 7.83221 E-012 & 7.83203 E-012 & 7.83202 E-012 \\
\hline
\end{tabular}

Table 7: Error of $u$ in solving Problem 3 with each proposed method, using $\nu=0.5$ and $\Delta t=10^{-3}$

\begin{tabular}{ccccc}
\hline Grid Size & FTCS & Crank-Nicolson & AI2*-AB3 & AM2*-AB3 \\
\hline $4 \times 4$ & 3.37083 E-007 & 3.78923 E-007 & 3.78238 E-007 & 3.78638 E-007 \\
$8 \times 8$ & 5.20385 E-008 & 8.58507 E-008 & 8.52775 E-008 & 8.56141 E-008 \\
$16 \times 16$ & 1.10676 E-008 & 2.09068 E-008 & 2.03598 E-008 & 2.06813 E-008 \\
$32 \times 32$ & N/A & 5.15543 E-009 & 4.61497 E-009 & 4.93273 E-009 \\
$64 \times 64$ & N/A & 1.24730 E-009 & 7.08456 E-010 & 1.02530 E-009 \\
\hline
\end{tabular}

Table 8: Error of $v$ in solving Problem 3 with each proposed method, using $\nu=0.5$ and $\Delta t=10^{-3}$

\begin{tabular}{ccccc}
\hline Grid Size & FTCS & Crank-Nicolson & AI2*-AB3 & AM2*-AB3 \\
\hline $4 \times 4$ & 1.55049 E-008 & 1.72357 E-008 & 1.72090 E-008 & 1.72265 E-008 \\
$8 \times 8$ & 2.94051 E-009 & 4.76637 E-009 & 4.73862 E-009 & 4.75699 E-009 \\
$16 \times 16$ & 6.41358 E-010 & 1.21990 E-009 & 1.19182 E-009 & 1.21046 E-009 \\
$32 \times 32$ & N/A & 3.03978 E-010 & 2.75867 E-010 & 2.94543 E-010 \\
$64 \times 64$ & N/A & 7.37489 E-011 & 4.56236 E-011 & 6.43088 E-011 \\
\hline
\end{tabular}

At a grid size of 16x16 cells, it is noticeable that the explicit scheme is no longer stable, as the error for $v$ unexpectedly returns an order of magnitude smaller than the other schemes. An increase in resolution confirms the instability, as the code fails to finish the following simulations. Both the implicit and IMEX schemes remain stable for the tested spatial resolutions, but AI $2 *-\mathrm{AB} 3$ shows signs of instability at a grid size of $64 \times 64$ cells. For the tested resolutions, $\mathrm{AM} 2 *-\mathrm{AB} 3$ provides slightly more accurate results than Crank-Nicolson while still treating the nonlinear term explicitly.

In addition to the numerical accuracy of the solution, another important consideration when implementing time integration schemes is the processing time. Where explicit methods are easy to code and optimize, and thereby inherently faster with naive implementations, IMEX and implicit schemes require careful code optimization to ensure good performance. While 
this investigation is not the focus of this work, a cursory comparison of the execution times of AM2*-AB3 and Crank-Nicolson algorithms for Problem 3 is presented in Table 9 for resolutions where the FTCS scheme is no longer stable. The last column shows the speedup achieved by the AM $2 *-\mathrm{AB} 3$ scheme over the corresponding Crank-Nicolson one. Initial and boundary conditions are taken from the experiment detailed in Tables 7 and 8.

Table 9: Execution times (in seconds) of Crank-Nicolson and AM2*-AB3 schemes for Problem 3 and speedup of AM2*-AB3 over Crank-Nicolson

\begin{tabular}{cccc}
\hline Grid Size & Crank-Nicolson & AM2*-AB3 & Speedup \\
\hline $32 \times 32$ & 152.376 & 33.194 & 4.59 \\
$64 \times 64$ & 7013.726 & 1387.785 & 5.05 \\
\hline
\end{tabular}

It should be noted that the results are obtained using straightforward, non-optimized implementations. The execution times of both IMEX methods are identical, since the implementation uses numerical constants $b$ and $c$ to control the integration scheme, as described in Eq. (21). Therefore, only the times for $\mathrm{AM} 2 *-\mathrm{AB} 3$ are shown.

\section{FINAL REMARKS}

This work compared some implementations of the IMEX method to the ones using a Euler explicit method (FTCS) and a Crank-Nicolson implicit method for the numerical solution of partial differential equations. The test cases are related to the time integration of the $1 \mathrm{D}$ and $2 \mathrm{D}$ Burgers' equation, which are simplified models in fluid dynamics to simulate both nonlinear advective and diffusive viscous processes.

Explicit schemes are simpler, but are not convenient for problems with fast time scales due to the small time steps required to ensure numerical stability, which may demand a high number of calculations in order to complete the simulation. Implicit schemes, on the other hand, are not convenient for problems with slow time scales, since these often require the solution of a nonlinear algebraic system at each time step by employing iterative schemes that may also demand high processing times. IMEX schemes can be useful for problems that have both fast and slow time scales, combining and taking advantage of explicit and implicit schemes. One-dimensional and two-dimensional test cases with exact analytical solutions were chosen, in order to compare the accuracy of the solutions obtained by the different numerical implementations corresponding to the proposed IMEX schemes.

The results show that IMEX methods can successfully solve Burgers' equation with accuracy comparable to a fully implicit method, even while treating the nonlinear term explicitly. Furthermore, such methods remain stable at higher resolutions than the explicit scheme, constituting an interesting alternative for these cases.

In this work, the focus was the accuracy obtained by the different IMEX schemes in these test cases, but the computational performance is still being investigated. Nevertheless, a preliminary comparison shows that IMEX schemes perform significantly faster than the CrankNicolson scheme while maintaining the same order of numerical accuracy in test cases that a fully explicit scheme could not solve. Code optimization is currently underway with a focus 
on some inefficient routines from standard libraries. A more comprehensive analysis of processing times, including comparisons with explicit schemes and analysis of stability regions to determine optimal time step sizes, will be shown in a future work.

\section{ACKNOWLEDGEMENTS}

The authors would like to thank the following Brazilian research support agencies: CAPES, CNPq, and FAPESP.

\section{REFERENCES}

Anderson, J. D., 1996. Discretization of partial differential equations. In Wendt, J. F., ed, Computational Fluid Dynamics: an Introduction, pp. 87-103. Springer.

Ascher, U. M., Ruuth, S. J., \& Wetton, B. T. R., 1995. Implicit-explicit methods for timedependent partial differential equations. SIAM Journal on Numerical Analysis, vol. 32, n. 3, pp. 797-823.

Bateman, H., 1915. Some recent researches on the motion of fluids. Monthly Weather Review, vol. 43, n. 4, pp. 163-170.

Burgers, J. M., 1948. A mathematical model illustrating the theory of turbulence. In von Mises, R., \& von Kármán, T., eds, Advances in Applied Mechanics, pp. 171-199. Elsevier.

Canuto, C., Hussaini, A., Quarteroni, A., \& Zang, T., 1987. Spectral Methods in Fluid Dynamics. Springer-Verlag.

Chapra, S. C., \& Canale, R. P., 2010. Numerical Methods for Engineers. McGraw-Hill.

Durran, D. R., \& Blossey, P. N., 2012. Implicit-explicit multistep methods for fast-wave-slowwave problems. Monthly Weather Review, vol. 140, n. 4, pp. 1307-1325.

Fletcher, C. A. J., 1983. Generating exact solutions of the two-dimensional Burgers' equations. International Journal for Numerical Methods in Fluids, vol. 3, n. 3, pp. 213-216.

Fornberg, B., \& Driscoll, T., 1999. A Fast Spectral Algorithm for Nonlinear Wave Equations with Linear Dispersion. Journal of Computational Physics, vol. 155, pp. 456-467.

Kadioglu, S. Y., \& Knoll, D. A., 2011. An IMEX Method for the Euler Equations that Posses Strong Non-Linear Heat Conduction and Stiff Source Terms (Radiation Hydrodynamics). In Schulz, H., ed, Hydrodynamics - Advanced Topics, pp. 293-318. InTech.

Kim, J., \& Moin, P., 1985. Application of a fractional-step method to incompressible NavierStokes equations. Journal of Computational Physics, vol. 59, pp. 308-323.

Kweyu, M. C., Manyonge, W. A., Koross, A., \& Ssemaganda, V., 2012. Numerical solutions of the Burgers' system in two dimensions under varied initial and boundary conditions. Applied Mathematical Sciences, vol. 6, n. 113, pp. 5603-5615.

Lemieux, J. F., Knoll, D. A., Losch, M., \& Girard, C., 2014. A second-order accurate in time IMplicit-EXplicit (IMEX) integration scheme for sea ice dynamics. Journal of Computational Physics, vol. 263, pp. 375-392. 
Liu, D., \& Wang, Y., 2015. High order numerical solutions to convection diffusion equations with different approaches. Journal of Applied \& Computational Mathematics, vol. 4, n. 2, pp. 208.

Mesinger, F., \& Arakawa, A., 1976. Numerical Methods Used in Atmospheric Models, Volume 1. International Council of Scientific Unions - World Meteorological Organization.

Nielsen, T. B., Fisher, T. C., \& Frankel, S. H., 2014. High-order implicit-explicit multi-block time-stepping method for hyperbolic PDEs. In 52nd Aerospace Sciences Meeting, AIAA SciTech Forum.

Srivastava, V. K., Tamsir, M., Bhardwaj, U., \& Sanyasiraju, Y. V. S. S., 2011. Crank-Nicolson scheme for numerical solutions of two-dimensional coupled Burgers' equations. International Journal of Scientific \& Engineering Research, vol. 2, n. 5, pp. 44-50.

Weller, H., Lock, S. J., \& Wood, N., 2013. Runge-Kutta IMEX schemes for the horizontally explicit/vertically implicit (HEVI) solution of wave equations. Journal of Computational Physics, vol. 252, pp. 365-381.

Zhu, H., Shu, H., \& Ding, M., 2010. Numerical solutions of two-dimensional Burgers' equations by discrete Adomian decomposition method. Computers \& Mathematics with Applications, vol. 60, n. 3, pp. 840-848. 